\title{
Foreword of the Special Issue on Motivation in Physical Education, Sport and Physical Activity and Health
}

\author{
by \\ Pedro Antonio Sánchez Miguel1, Diana Amado
}

Following the growing concern of health specialists over physical activity levels in a population with high rates of sedentary behaviors, the aim of this Special Issue is to emphasize the importance of motivation to create and maintain healthy habits.

In this regard, the articles included in this Special Issue are classified in four domains where the study of motivation is crucial: physical education, sport, physical activity and health. In recent years, there have been lots of scientific studies that attempted to assess motivation in the aforementioned contexts, increasing their presence in journals of sports sciences, education, medicine, psychology, multidisciplinary areas, etc., contributing also to a greater readership of periodicals where these investigations were published.

Regarding the physical education context, there is currently a greater interest of professionals involved in the domain of educative psychology for the research of students' motivation, as well as the utility given by pupils to the different subjects taught. The physical education context is considered an excellent tool to promote and develop knowledge and positive attitudes towards the practice of physical activity, and increase adherence to these activities. Thus, maladaptive consequences that emerge during the adolescence might be avoided.

In accordance with this, the Special Issue includes four interesting articles that deal with motivation in physical education, the use of teaching games to promote student's motivation in physical education, the relationships between physical activity levels, self-identity, body dissatisfaction and motivation, and changes in self-determined motivation in order to induce engagement in physical education.

Respecting the sport domain, it is well known that psychological variables are of great relevance to sport, performance and also to the creation of adaptive behaviors within the sport, regardless the sports level. In this context, the Special Issue includes a mixed intervention program (cognitive-motivational) in order to assess satisfaction and performance. Moreover, a manuscript showing the prevalence and covariates of potential doping behavior in kickboxing is included. Furthermore, two more articles dealing with mental strategies to predict performance and satisfaction with performance in soccer players and the importance of the context in goal pursuit, burnout and motivation in female athletes from different sports, are presented. Finally, our readers may get acquainted with an intervention program with coaches to improve satisfaction of basic psychological needs and self-determination levels in young soccer players.

Lastly, as we have previously indicated, motivation promotes changes in behaviors and increases awareness of the importance of physical activity for health. Thus, some articles included in the Special Issue

1 - Faculty of Teaching Training, University of Extremadura (Spain).

2 - Faculty of Juridical and Social Sciences, Rey Juan Carlos University (Spain). 
deal with motivational antecedents of well-being and health related behaviors in adolescents, intervention programs to optimize motivation and improve eating habits, as well as evaluate the influence of goal contents on exercise addiction, examining the mediating effect of passion for exercise.

Finally, we may conclude this foreword indicating our desire that the manuscripts included in the current Special Issue will let improve scientific knowledge of the readers about the motivational aspects in the previously suggested domains, associated to the practice of physical activity of the population. Moreover, we would like to stimulate the scientific community to continue with this line of investigation that emerges from sports psychology and is applied in physical activity and sports sciences.

Pedro Antonio Sánchez Miguel

Diana Amado

Guest editors of the Special Issue 